\title{
Treatment of Acid Orange 74 Wastewater and Sugar Wastewater by Low Cost Adsorbents
}

\author{
S.S.V. Harsha Madiraju ${ }^{1}$, Yung-Tse Hung ${ }^{1, *}$, Howard H. Paul ${ }^{2}$ \\ ${ }^{1}$ Department of Civil and Environmental Engineering, Cleveland State University, Cleveland, Ohio 44115, USA \\ ${ }^{2}$ Department of Information Systems, Cleveland State University, Cleveland, Ohio 44115, USA.
}

\section{ART ICLE DETAILS}

\section{Article history:}

Received 15 June 2018

Accepted 24 June 2018

Available online 28 October 2018

\section{Keywords:}

Acid Orange 74

Dye wastewater

Color Removal

\begin{abstract}
A B S T R A C T
This study shows the treatment of combined binary mixture of Acid Orange 74 and sugar wastewater with peanut hull and orange peel as low-cost adsorbents. The performance of a combined adsorptionmicrofiltration process for the color removal is measured and compared using transmittance and absorbance indices at mechanical shaker mix level. This selected treatment procedure is among one of the most economical treatment alternatives to all technologies present now. The parameters studied during this research are analyzed using Langmuir and Freundlich isotherm models on low cost adsorbents. Conclusive results after the treatment are indicated in this paper at their optimum dosages and sizes. This treatment method is applicable in the actual conditions at territory treatment stage.
\end{abstract}

\section{Introduction}

The discharge of wastewater from the textile industries is considered among one of the most polluting sources of environment [1]. The fabric creating the textiles requires massive amounts of energy, uses vast constituents of resources, and often leaves huge volumes of wastes. The process of making textiles and the way pollutants are used can often has an enormous impact on environment. Stages like washing, leaching, and dyeing in the textile industries involves in usage of large amounts of water which is drained as wastewater. This production of textile industries is directly proportional to population growth and increase in usage than the past [1].

Sugar industry is one of the most agriculture-based industry and has a significant impact on nation's economy. Sugar is either used in the power form or in the production of candies, ice creams, bakery items etc., [2]. As the usage is increased creates a necessary for the increase in production which ultimately increase the pollution. This industry has an impact on ambient air, water, and soil in one or the other way [2].

Acid orange 74 (Fig. 1) has C.I. number 18745 with molecular structure of single azo, metal complexes. Its molecular formula is $\mathrm{C}_{16} \mathrm{H}_{12} \mathrm{~N}_{5} \mathrm{NaO}_{7} \mathrm{~S}$ with 441.35 formula weight. It is an orange, deep brown powder. It is soluble in water for orange, soluble in ethanol, slightly soluble in soluble fiber element, conc. sulfuric acid to dark yellow, diluted into orange; in $10 \%$ of sodium hydroxide solution for orange. It is used for wool, polyamide fiber and silk dyeing and printing directly, also used in leather and electrochemical aluminum color. Especially for the influential product and the wool carpet dyeing $[3,4]$.

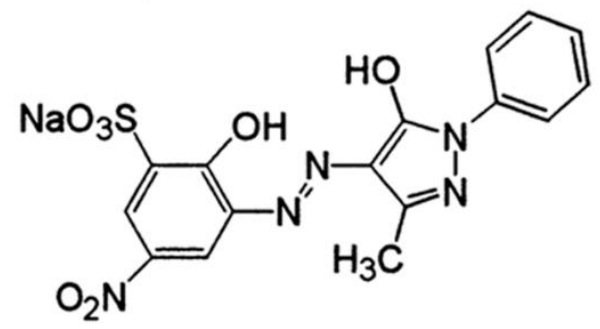

Fig. 1 Molecular structure of Acid Orange 74

\section{Experimental Methods}

\subsection{Adsorbents}

\subsubsection{Peanut Hull}

The peanut hull used in this research is taken out from the peanuts purchased at local grocery store. They are washed with distilled water thoroughly and oven dried at $70{ }^{\circ} \mathrm{C}$ for $24 \mathrm{~h}$ [5]. Then peanut hull is grounded into powder and sieved into five varied sizes (powder retained on 3327-2380 $\mu \mathrm{m}, 2380-2362 \mu \mathrm{m}, 2362-600 \mu \mathrm{m}, 600-425 \mu \mathrm{m},<425 \mu \mathrm{m}$ ). Single dosage with five varied sizes is used in this research. These powdered peanut hulls are used as adsorbent to treat combined wastewater through adsorption [6].

\subsubsection{Orange Peel}

The orange peel used in this research is taken from the oranges purchased at local grocery store. They are washed with distilled water and oven dried at $70^{\circ} \mathrm{C}$ for $24 \mathrm{~h}$ and grounded into powder as like peanut hull but sieved into only one size $(425 \mu \mathrm{m})$. Single size with varied dosage levels are used in this research [7-9].

\subsection{Datum}

The powdered activated carbon from DARCO with HDC grade is used in this research as the reference adsorbent. The experiments in this research are started with trial and error process to choose the concentrations and dosages [10]. Then started experiments with activated carbon at ideal conditions, which are used as a datum to compare with primary adsorbents.

\subsection{Combined Binary Wastewater Mix}

The mother samples of combined binary wastewater mix are prepared in the ideal laboratory conditions with Acid Orange 74 and Sugar Powder [11]. The samples with specific concentrations of binary wastewater mix are prepared in equal volumes in numerous samples. The samples are prepared in a batch to get treated with low-cost adsorbents.

\subsection{Categorization of Dye Concentration}

The dye concentrations used in this research are categorized into four types. They are low concentration, lower medium concentration, upper medium concentration and high concentration. Based on trial tests performed with dye concentrations these are categorized [12]. For Acid Orange 74 the concentrations taken in the respective category are given in Table 1. 
Table 1 Concentrations of Acid Orange 74 considered

\begin{tabular}{ll}
\hline Category & Concentration \\
\hline Low Concentration & $50 \mathrm{ppm}$ \\
Lower Medium Concentration & $100 \mathrm{ppm}$ \\
Upper Medium Concentration & $150 \mathrm{ppm}$ \\
High Concentration & $200 \mathrm{ppm}$ \\
\hline
\end{tabular}

\subsection{Method}

This research follows batch mode analysis where in each sample of size $100 \mathrm{~mL}$ is prepared by diluting the dye (Acid Orange 74) stock solutions and adsorbent stock solutions in aqueous form to ensure proper mixing and minimal losses. The desired concentrations achieved using mechanical shaker mix followed by $24 \mathrm{~h}$ settlement. The supernatant achieved is used to analyze the transmittance and absorbance. Then they are treated with low cost adsorbents with a multistage treatment process with adsorption followed by microfiltration. The whole experiment is under ideal laboratory conditions. The equilibrium adsorption capacity is then calculated.

\section{Results and Discussion}

The major parameter used in this research to distinguish and compare the treatment capacity of the low-cost adsorbents is transmittance (\%). The amount of light that can pass through a substance is called transmittance. Distilled water, which is manufactured in the laboratory is considered as reference due to its 0 absorbance (minimum) and 100\% (maximum) transmittance [13]. It can also be defined as the ratio of intensity of incident light to transmitted light. It is measured in $\%$.

The powdered orange peel is first considered to treat the combined wastewater as a low-cost adsorbent. Powdered orange peel is considered in four different dosages with same sieve size [14]. The powder which is passed through $425 \mu \mathrm{m}$ sieve is used as adsorbent. The dosage is in ascending order from $0.5 \mathrm{~g}$ to $2 \mathrm{~g}$ at equal intervals of $0.5 \mathrm{~g}$ per $100 \mathrm{~mL}$ of combined wastewater. The results are analyzed with total 936 sample runs to estimate the efficiency of orange peel to estimate the treatment capacity. The transmittance when treated with orange peel powder at low concentration and high concentration are plotted in Figs. 2 and 3.

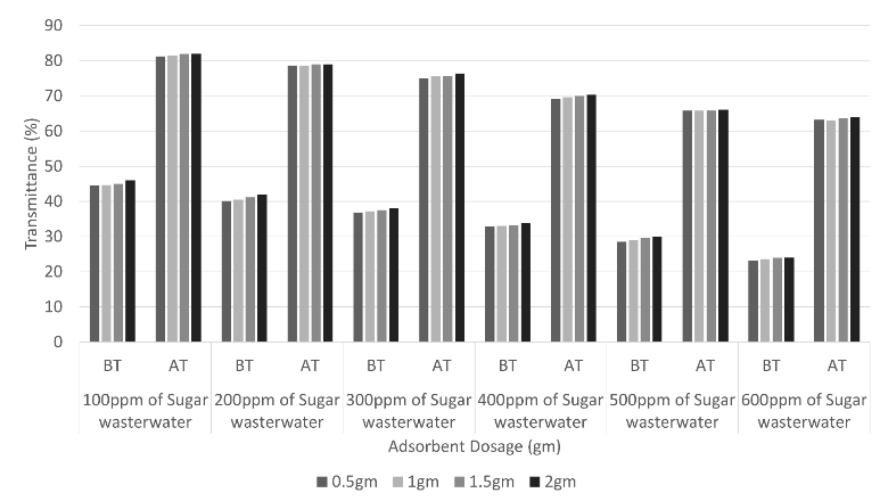

Fig. 2 Transmittance of Acid Orange 74 (50 ppm) with orange peel

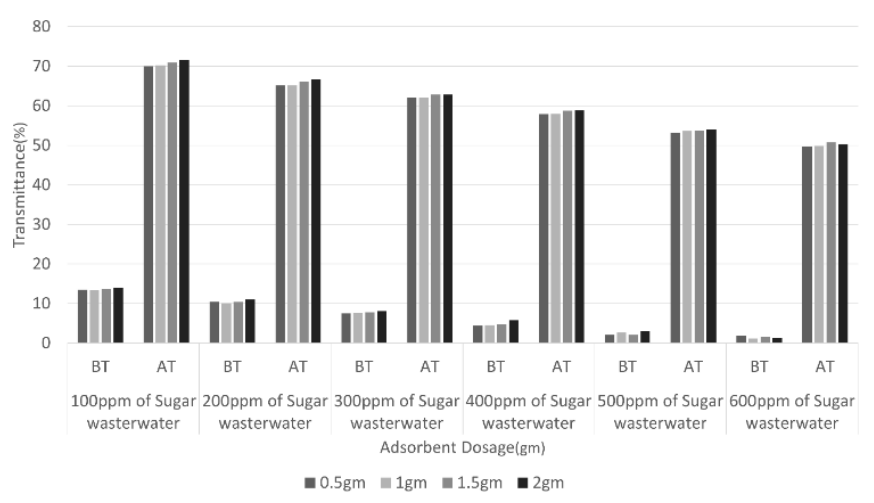

Fig. 3 Transmittance of Acid Orange 74 (200 ppm) with orange peel

The peanut hull is second considered to treat the combined wastewater as a low-cost adsorbent. Peanut hull is considered in four different dosages with five different sieve sizes. The peanut hull in the sieve range of 3327-2380 $\mu \mathrm{m}, 2380-2362 \mu \mathrm{m}, 2362-600 \mu \mathrm{m}, 600-425 \mu \mathrm{m},<425 \mu \mathrm{m}$ https://doi.org/10.30799/jacs.188.18040304 are added at a constant dosage of 2 g per $100 \mathrm{~mL}$ of combined wastewater. The results are analyzed with total 936 sample runs to estimate the efficiency of peanut hull to estimate the treatment capacity. The transmittance when treated with peanut hull at low concentration and high concentration are plotted in Figs. 4 and 5.

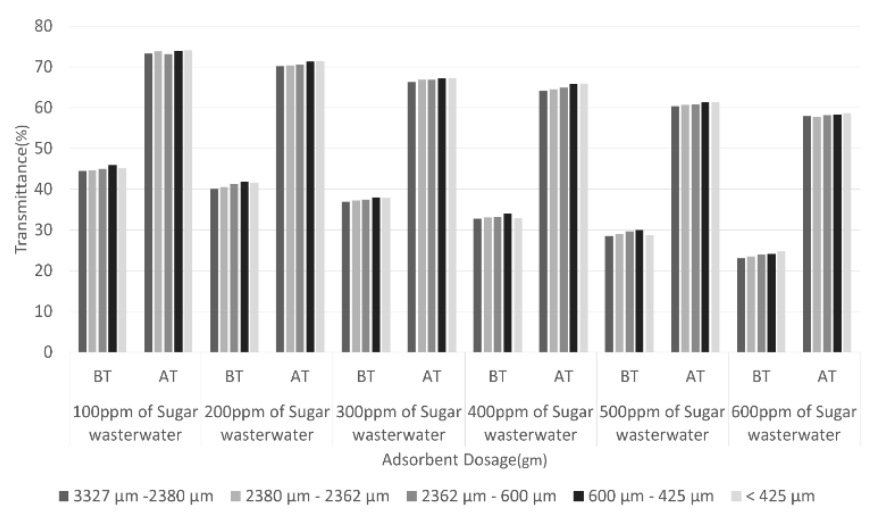

Fig. 4 Transmittance of Acid Orange 74 (50 ppm) with peanut hull

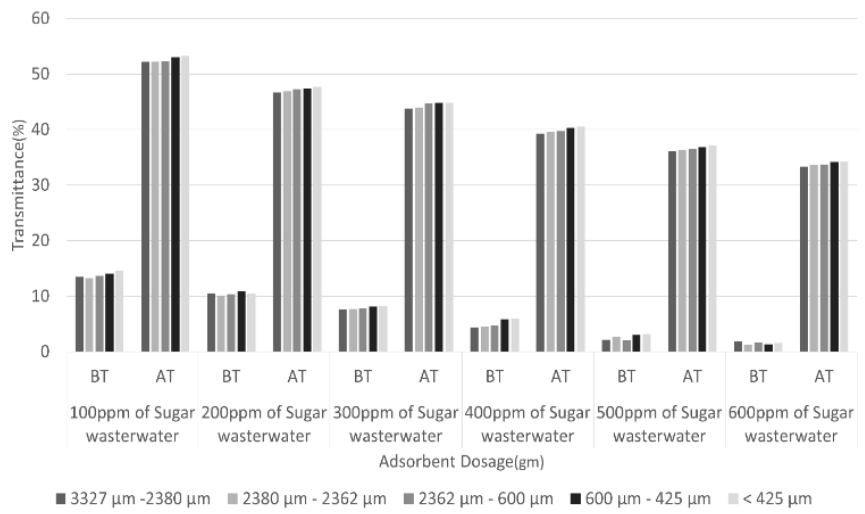

Fig. 5 Transmittance of Acid Orange 74 (200 ppm) with peanut hull

\subsection{Adsorption Isotherms}

The linearized isotherms are studied at the equilibrium to estimate the best model fit after the treatment with low cost adsorbents [15]. The equilibrium studies are performed by adding orange peel powder and peanut hull at considered dosages sizes to $100 \mathrm{~mL}$ of combined wastewater with 50 ppm, 100 ppm, 150 ppm, 200 ppm of Acid Orange 74 and $100 \mathrm{ppm}, 200 \mathrm{ppm}, 300 \mathrm{ppm}, 400 \mathrm{ppm}, 500 \mathrm{ppm}, 600 \mathrm{ppm}$ concentrations of sugar wastewater.

The linearized Langmuir isotherm model (Fig. 6) found to be best fit for the adsorption on orange peel. The linearized Langmuir isotherm is represented with Eq.(1),

$$
\frac{1}{q e}=\frac{1}{q m k a} * \frac{1}{C e}+\frac{1}{q m}
$$

The linearized Freundlich isotherm model (Fig. 7) found to be best fit for the adsorption on peanut hull. The linearized Freundlich isotherm is represented with Eq.(2),

$$
q=k * C^{1 / n}
$$

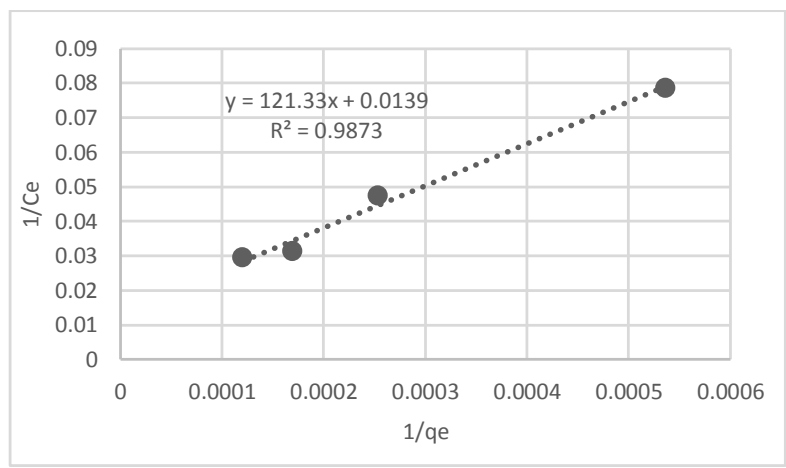

Fig. 6 Langmuir isotherm model of Acid Orange 74 adsorption on Orange pee 


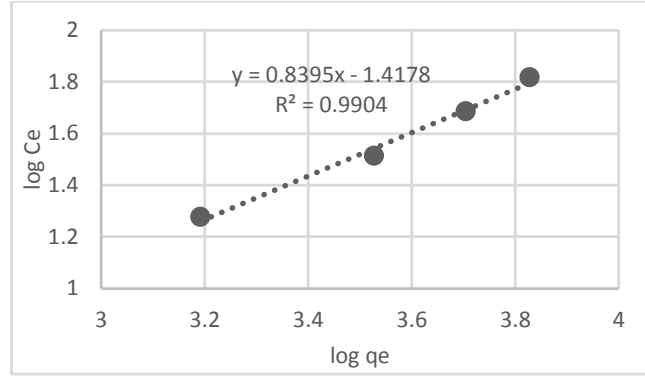

Fig. 7 Freundlich isotherm model of Acid Orange 74 adsorption on peanut hull

Table 2 Best fit isotherms for Acid Orange 74 at optimum dosage and size

\begin{tabular}{llll}
\hline Adsorbents & Isotherm & Linear equation & $\begin{array}{l}\text { Coefficient of } \\
\text { determination }\end{array}$ \\
\hline Orange Peel & Langmuir & $\mathrm{y}=121.33 \mathrm{x}+0.0139$ & 0.9873 \\
Peanut Hull & Freundlich & $\mathrm{y}=0.8395 \mathrm{x}-1.4178$ & 0.9904 \\
\hline
\end{tabular}

The organic carbon remained after purging the acidified sample with gas is called as non-purgeable organic carbon. The NPOC is also an additional parameter calculated to estimate its presence after the treatment is calculated. The non-purgeable organic carbon for the high concentration Acid Orange $74(200 \mathrm{ppm})$ with increasing sugar wastewater concentration (mg/L) is plotted in Fig. 8.

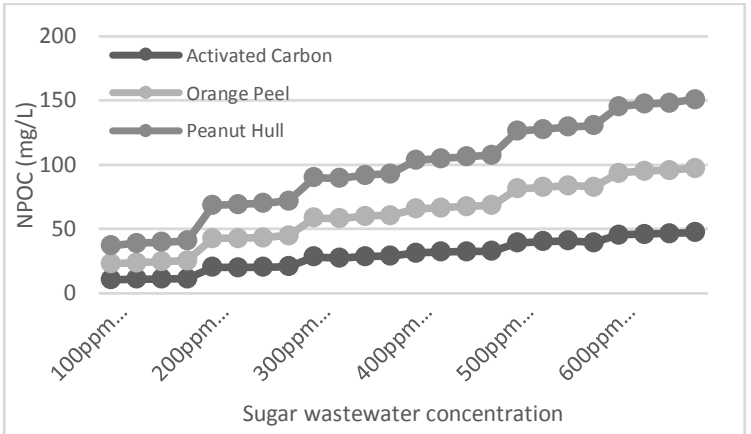

Fig. 8 Comparison of NPOC at high concentration Acid Orange 74

The phenomenon observed after treatment was increase in rate of adsorption due to increase in surface area, which had impact on treatment capacity. The $\mathrm{pH}$ is very important in adsorption process especially dye adsorption. The rate of adsorption and magnitude of electrostatic charges due to ionized dye molecules is controlled by level of $\mathrm{pH}$, preferably neutral/medium. $\mathrm{pH}$ is inversely proportional to anionic dye adsorption and directly proportional to cationic dye adsorption. The initial $\mathrm{pH}$ of the combined wastewater samples is in the neutral range of 6 to 9. During the experiments the contact time of the combined wastewater samples with the adsorbent was $24 \mathrm{~h} \pm 1 \mathrm{~h}$. The whole experiment for a set of samples is being completed in not more than $48 \mathrm{~h}$. The effect of oxidation of dye increases as the duration of experiment increases [16].

\section{Conclusion}

This treatment was mainly focused on color removal of Acid Orange 74 at four different considered concentrations with a combined binary mixture of sugar wastewater at ideal laboratory conditions with low cost adsorbents orange peel, peanut hull considered at assorted sizes and dosages with activated carbon as datum. The maximum transmittance achieved at the optimum dosage of orange peel is up to $82.03 \%$ and the peanut hull up to $74.23 \%$. The minimum NPOC values achieved after the treatment with orange peel and peanut hull are $35.06 \mathrm{mg} / \mathrm{L}$ and 37.86 $\mathrm{mg} / \mathrm{L}$ respectively at $100 \mathrm{ppm}$ sugar concentration. The linearized Langmuir isotherm model is found to be best fit for the adsorption on orange peel and the linearized Freundlich isotherm model is found to be best fit for the adsorption on peanut hull. The $\mathrm{pH}$ adjustment is neglected as it is already in neutral range. The experiment is performed at room temperature in order to eliminate its effect.

\section{Acknowledgments}

The authors thank Cleveland State University for providing support and essential research facilities.

\section{References}

[1] Md. Mahmudur Rahman, Bari Quazi, Treatment of textile wastewater using laboratory produced activated carbon, Environ. Sustain. Develop. 2011 (2011) 30727-1-7.

[2] Poddar, Pradeep Kumar, Sahu, Omprakash, Quality and management of wastewater in sugar industry, Appl. Water Sci. 7 (2014) 461-468.

[3] Marius Sebastian Secula, Benoît Cagnon, Igor Crețescu, Mariana Diaconu, Stelian Petrescu, Removal of an acid dye from aqueous solutions by adsorption on a commercial granular activated carbon: equilibrium, kinetic and thermodynamic study, Sci. Study Res. 12(4) (2011) 307-322.

[4] G.B. Oguntimein, Textile dye removal using dried sun flower seed hull a new low cost bio sorbent: equilibrium, kinetics and thermodynamic studies, Adv.Res. Textile Eng. 1(1) (2016) 1-7.

[5] N.A. Taha, A. El-Maghraby, Characterization of peanut hulls and adsorption study on basic dye: isotherm and kinetic analysis, International J. Innovat. Res. Tech. Sci. 2 (2011) 9-18.

[6] Sunil Kumar, V. Gunasekar, V. Ponnusami, Removal of methylene blue from aqueous effluent using fixed bed of groundnut shell powder, Jour. Chem. 2013 (2013) 259819-1-5.

[7] F.B. Abdurrahman, M. Akter, M. Zainal Abedin, Dyes removal from textile wastewater using orange peels, International Jour. Sci. Technol. 2(9) (2013) 47-50.

[8] A.G. El-Said, A.M. Gamal, Potential application of orange peel (op) as an ecofriendly adsorbent for textile dyeing effluents, J. Textile Apparel Tech. Manag. 7(3) (2012) 1-13.

[9] R.S. Mane, V.N. Bhusari, Removal of color (dyes) from textile effluent by adsorption using orange and banana peel, Int. J. Eng. Res. Appln. 2(3) (2012) 1997-2004.

[10] Y. Majedi, E. Alhilali, M. Al Nehayan, A. Rashed, S. Shwkat Ali, et al., Treatment of dye-loaded wastewater with activated carbon from date palm leaf wastes, The 4th World Sustainability Forum, Canada, 2014, pp.1-12.

[11] E. Mourid, M. Lakraimi, E. El Khattabi, L. Benaziz, M. Berraho, Removal of textile dye acid green 1 from wastewater by activated carbon, Jour. Mater. Environ. Sci. 8(9) (2017) 3121-3130.

[12] Saad Saeed, Sadia Khan, Sana Saeed, Rafiullah Khan, Removal of dyes from textile waste water using adsorption by activated carbon of rice husk, Int. J. Innovat. Sci. Res. 17 (2015) 191-196.

[13] T.M. Al Khusaibi, J.J. Dumaran, M. Geetha Devi, L. Nageswara Rao, S. Feroz, Treatment of dairy wastewater using orange and banana peels, Jour. Chem. Pharm. Res. 7(4) (2015) 1385-1391.

[14] Indira Khatod, Removal of methylene blue dye from aqueous solutions by neem leaf and orange peel powder, Int. Conf. Global Scenario Environ. Energy 5(2) (2013) 572-577.

[15] Ayesha Wasti, M. Ali Awanb, Adsorption of textile dye onto modified immobilized activated alumina, Jour. Assoc. Arab Univ. Basic Appl. Sci. 20 (2016) 26-31.

[16] H. Benaïssa, Removal of acid dyes from aqueous solutions using orange peel as a sorbent material, Int. J. Env. Pollut. 34 (2008) 71-82. 